ARTICLE

\title{
Study on Cognitive Optical Network Structure and Self-optimization with the Application of Artificial Intelligence Technology
}

\section{Shengzhe Liang*}

Rutgers, The State University of New Jersey, New Brunswick, US

\begin{tabular}{ll}
\hline ARTICLE INFO & ABSTRACT \\
\cline { 2 - 2 } $\begin{array}{l}\text { Article history } \\
\text { Received: } 6 \text { January } 2020\end{array}$ & $\begin{array}{l}\text { Cognitive optical network is the intermediate to combine artificial intel- } \\
\text { ligence technology with network, and also the important network tech- } \\
\text { nelogy to promote network intelligence level constantly. In the paper, it } \\
\text { analyzes the cognitive optical network structure with the application of } \\
\text { artificial intelligence technology by starting from the basic conditions of } \\
\text { Accepted: } 9 \text { April } 2020\end{array}$ \\
cognitive network and cognitive optional network on the basis of fully \\
understanding the connotation of cognitive network and cognitive optical \\
network, and explores its self-governance functions, so as to better realize \\
the self-optimization and self-configuration of network.
\end{tabular}

Cognitive optical network

Network structure

Self-optimization

\section{Introduction}

$\mathrm{A}$ $s$ the development level of information technology is promoted, internet traffic has shown a substantial increase over the years, reached to 2ZB in 2019, and increased the standard of network bandwidth demands. Since traditional network transmission technologies cannot meet the network operating demands, it is certain that new network transmission technologies will be generated as the replacement. Therefore, optical link coding technologies, super-channel technologies, and optical modulation technologies have been practiced in network transmission at different levels over the years. These new technologies are mainly deployed in the original optical network, and can largely increase the complicity of network environment.
Meanwhile, the same optical fiber will exert direct influence on network transmission quality and transmission speed after being shared by different systems due to the increase in transmission capacity and the requirements of transmission speed. However, optical network is an autonomous network that is easy to maintain and manage, with low manual intervention demand, and can meet the demand of the current network transmission for intelligent management. On this basis, cognitive optical network has emerged in recent years. It combines network characteristics with artificial intelligence technology advantages, and is equipped with self-governance function. It is of great significance to improve network transmission quality and service quality by exploring how to solve the aforementioned problems with artificial intelligence technology and realize self-optimization.

\footnotetext{
*Corresponding Author:

Shengzhe Liang,

Male, a student majored in Computer,

The State University of New Jersey, 57 US Highway 1,New Brunswick, NJ 08901-8554;

E-mail: shengzhe.liang@rutgers.edu.
} 


\section{Cognitive Network and Cognitive Optical}

\section{Network}

\subsection{Overview to Cognitive Network}

Cognitive network can not only detect network environment, but also make intelligent plan for network by making full use of the local automatic network environment adaptability, and constant learning capacity. Besides, it can also make correct decisions to realize end-to-end objectives, and then lay a good development foundation for the follow-up dynamic changes and dynamic adjustment of network. Cognitive network is equipped with cognitive functions and learning functions. The most basic action to carry out cognitive network will form a complete cognition cycle, which includes five modules, i.e., Observe, Plan, Act, Orient, and Decide. In respect of Observe, it mainly adopts monitoring equipment in the real-time observation of network environment and network state, summarize the acquired data and provide basis for the Plan, while using the learning module to store the acquired data. In the process of Orient, suitable action mode should be selected by combing with the actual conditions about the background of observed information. After entering the phase of Decide, the cognitive network will formulate end-to-end system objective based on the actual act model selected and the plan achievements, and confirm suitable act in the end ${ }^{[1]}$. Finally, the link of Act is to ensure the execution of final decision in the network. Specific cycle process is as shown in Figure 1. This cycle can process new events in the network by constant cycle, and based on this characteristic, cognitive network is also called as autonomous network, and can optimize and configure as per network environment and network dynamics, and realize self-healing.

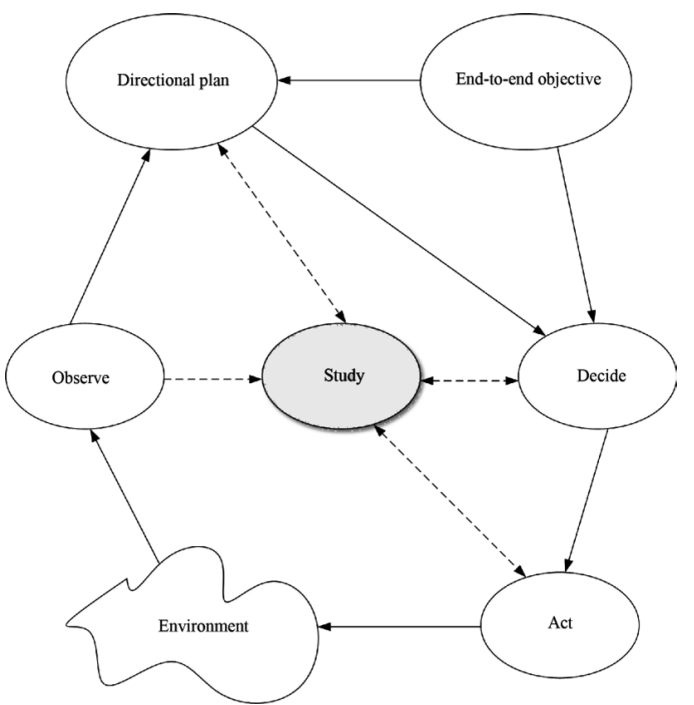

Figure 1. Schematic Diagram of Cognitive Network Cycle

\subsection{Overview to the Cognitive Optical Network}

Cognitive optical network has been the new direction of network technology studies over the years. Cognitive Heterogeneous Reconfigurable Optical Network Project (CHRON) funded by the European Union is currently the most successful research achievement at home and abroad, and its main objective is to develop cognitive optical network, provide support to control and manage the next generation of heterogeneous and optical network, and then become one of the major support technologies for the future development of internet. Cognitive Heterogeneous Reconfigurable Optical Network Research Project has completed the experimental research, and built intensive network architecture based on the research objective. Specific structure conditions are as shown in Figure 2. As can be seen from the figure, the project research is based on the deformation of distributed and intensive structures, but it is focused on the application of intensive type in test platforms ${ }^{[2]}$. As the most important part of the structure, cognitive decision system (CDS) exerts the effect of network event processing and the required management of network transmission, while network element is the key to ensure that cognitive actions can take effect in network operation. It is requested to consider the real-time status of network operation in structure architecture, and make full use of the previous knowledge to optimize network performance.

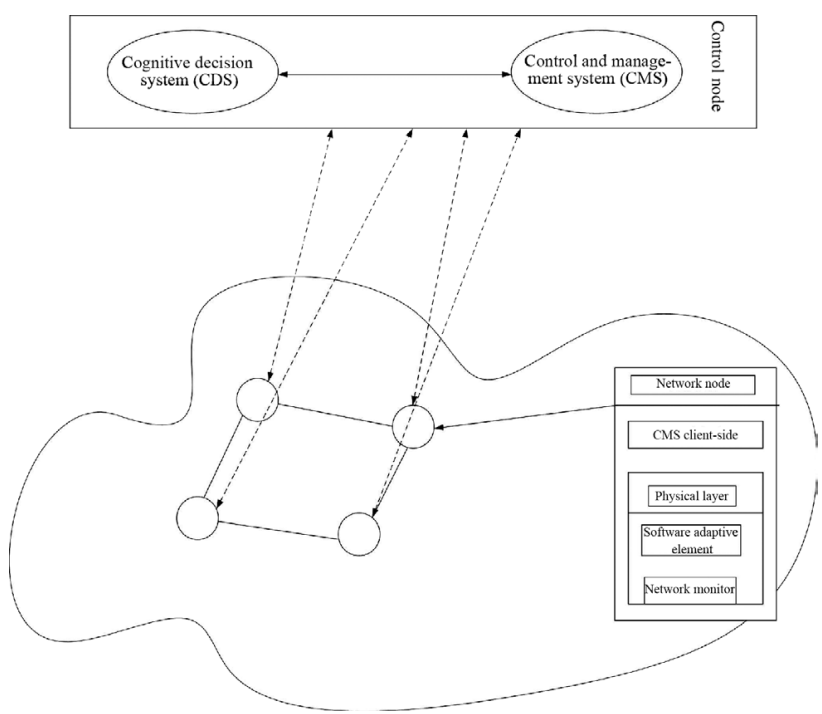

Figure 2. Cognitive Optical Network Structure Chart

Cognitive decision system (CDS) mainly exerts the effect of collecting monitoring information in network and sending management and control decisions to all cognitive decision systems (CDS) based on the control system and management system, while the self-optimization and 
self-configuration of cognitive optical network are realized by extensively collecting network dynamic operating conditions via cognitive decision system, sending decisions to cognitive decision system (CDS), and activating self-adaptive elements.

\section{Key Technologies and Structure of Cogni- tive Optical Network with the Application of Artificial Intelligence Technology}

The compositions and structures of cognitive optical network mainly include five aspects, i.e., intelligent monitoring system, cognitive decision system, cognitive control system, cognitive study, the dynamic adjustment of structure and automatic configuration respectively. Although each system has different functions and different divisions of labor in the cognitive process, all parts are closely connected, and will affect the function of the cognitive optical network, in case of any missing parts. Specifically speaking, the structural conditions and key technical analysis of all parts are as shown below:

\subsection{Intelligent Monitoring System}

The main function of intelligent monitoring system is network environment monitoring, and is equivalent to the observation mentioned above. Advanced optical performance monitoring technologies are used in monitoring, in respect of the physical layer. Such technology is equipped with digital signal processing function, and can provide the required feedback signal to the monitoring system, and then give comprehensive feedbacks about network damage conditions or signal defects, so that the cognitive optical network can recognize fast and make plans, realize autonomous optimization and configuration, and rebuild network operating environment ${ }^{[3]}$. In order to meet the demand of network users and the requirements put forward for service quality, a core module is set in the intelligent monitoring system-optical signal performance detection module, which can transmit the acquired information contents to network management and network decision system, and then express that the system has received information parameters provided by electric fish of the optical field in combination with the optical communication system and its corresponding receiver. Besides, channel distortion also regards data model as the supplementary algorithm of the electric field, to acquire real-time monitoring on the link damage information, and finally complete the automatic compensation of the electric field, and all the confirmed linear optical channel parameters, including polarization mode dispersion and dispersion, etc. are within the scope of isostatic compensation. Besides, intelligent monitoring system can also monitor transmission parameters, such as the polarization rotation parameters.

\subsection{Cognitive Decision System}

As the most important part of cognitive optical network, cognitive decision system works as the brain of cognitive optical network, to make decisions, transmit to the control and management links, etc., and then realize the self-optimization and self-configuration of cognitive optical network. In system operation, it will simultaneously analyze the monitoring information and the knowledge base, and put the currently acquired observation information in previous knowledge experience for specific analysis, and then carry out comprehensive analysis and judgment based on the occurred events and current conditions, and formulate the best processing schedule, so as to realize the end-toend objective effectively ${ }^{[4]}$. In the operation process of cognitive optical network, service and flow demand is the most basic supply of cognitive decision system. The cognitive optical network can seek for similar previous scenes and the decisions made thereby in the knowledge base based on the current network operating conditions during the operation, and then judge and make decisions based on the current operating conditions, store the analysis in the learning module, and improve learning capacity. Besides, it can also directly influence the future action of cognitive decision system, and then realize the end-to-end objective by network element adjustment based on the control and management system, and always realize the optimization of network transmission.

As can be seen from the operation and effect of cognitive decision system, it is closely related to network monitoring system and control system, and can form completed cognition cycle with two systems, while exerting its effect. Meanwhile, the cognitive optical network also has a specific knowledge base, which can search for information generated in all cognitive processes. It is generally arranged in the learning module, and will be updated in real time ${ }^{[5]}$. Therefore, a general database and specific database have also been formed in the cognitive optical network, and these two items can be activated and then implement information retrieval simultaneously in the cognitive process. The cognitive decision system can make decisions based on the current network operating conditions, and send instructions to network elements via the control system, and then network elements will complete decisions made by the cognitive decision system as the basic executing unit. In 
order to prevent network transmission quality from being influenced in the process, different routes can be used when setting new channels.

\subsection{Cognitive Control System}

Cognitive optical network mainly adopts distributed type as the main mode of management and control, but private or independent agreement contents will be increased in the construction of control system due to the difference in technologies, equipment and manufacturers, and such condition will largely increase the difficulty of network control, and influence the utilization efficiency of network resources. Therefore, the difference in the transmission quality and service quality incurred by the difference in transmission technologies and service demands should be considered in the development of cognitive optical network, to build a highly heterogeneous network operating environment ${ }^{[6]}$. There are domestic and foreign practices in this direction, such as GMPLS protocol family ASON (Automatically Switched Optical Network), GMPLS/PCE network control panel and SDN/Openflow SDON (Software Defined Optical Network), which have taken these heterogeneous conditions into considerations, optimized and adjusted the optical network structure accordingly, and then ensured that the control panel can be self-optimized based on actual network conditions, while executing the decisions made by the cognitive decision system.

\subsection{Cognitive Study Structure}

The intelligent characteristics of cognitive optical network can be mainly reflected by cognitive study, which will take effect in the operation of intelligent monitoring system. The study can be specific to network operation standard requirements, cognitive process plan, cognitive decisions and other series of process, and can ensure the real-time updating of knowledge base, apply the learned contents to handle network events in real time, and complete the target task of cognitive decision system. Cognitive study is mainly supported by machine study, and the common method is intelligent algorithm. Machine study is also called as case-based reasoning, which will associate with similar previous scenes and experience by reasoning the acquired knowledge experience, while solving new problems, and then adjusting parameters based on changes of the condition, and as processed events are increased, cases in the knowledge base will also increase constantly, so richer experience can be acquired while handling network events, and then it will improve the capacity of the system to solve problems ${ }^{[7]}$. Meanwhile, the system can also predict the future development conditions of network transmission by summarizing previous experience, so as to make predictable decisions and decrease the direct influence incurred by network transmission.

\subsection{Dynamic Adjustment and Automatic Configu- ration of Structure}

Cognitive optical network emphasizes on realizing endto-end objective, and should always regard the objective as the center to adjust parameters and configurations in the cognitive process and the decision execution process, and ensure that construction characteristics of physical layer and parameters of the network layer can meet all end-to-end objective requirements at all times ${ }^{[8]}$. Dynamic adjustment of structure and automatic configuration is the main approach to realize the function, and can carry out operations by software programming or software self-customization module.

\section{Conclusion}

To sum up, cognitive optical network is of important value and significance to the development of network intelligence as the new topic and new direction of research on network technology over the years, but current researches are still in the initial phase, and lack of experience in sorting out the structure of cognitive optical network and the corresponding design. We should cognize the connotation, function and structure of cognitive optical network correctly, understand its composition structure, define the specific reflection of intelligence based on the analysis, so that we can focus on the intelligent function and intelligent service of cognitive optical network in future development, perfect technical system, and then provide a solid guarantee for the improvement of network service quality and the transmission quality level. This paper still has deficiencies in simple analysis, and merely discusses how to realize the self-optimization of cognitive optical network and its specific structure. It is hoped that we can summarize research achievements constantly in future research, deepen research contents, and improve research depth and width.

\section{References}

[1] Huang Haiqing, and Li Weimin. Research on Cognitive Optical Network Structure Based on Artificial Intelligence Technology [J]. Optical Communication Technology, 2016, 40(5):15-18.

[2] Fu Dong. Research on the Perceptive Transmission Technology of Cognitive Optical Network [D]. Sich- 
uan: University of Electronic Science and Technology of China, 2019.

[3] University of Electronic Science and Technology of China. A Master-slave Signal Transmission Method in Cognitive Optical Network: CN201710959557.1 [P].2018-01-23.

[4] Ruan Xiaogang, Pang Tao, and Yu Jianjun. Phototaxis Control of Robot Based on the Neural Network Cognitive Mechanism of Boltzmann Machine [J]. Control and Decision-making, 2014, (12):2189-2194.

[5] Shenyang Aerospace University. A Deep Belief Network Intelligence Extraction Device in Cognitive Information Confrontation: CN201821413847.2
[P].2019-04-23.

[6] Wang Qian. "The Light of Reason" and "Experience Network"-Comparison of Two Cognitive Modes [J]. Journal of Dialectics of Nature, 2018, 40(12):94-100.

[7] Wei Jie. A Holographic 3D Interactive Display System Based on Artificial Intelligence Visual Recognition Technology: CN201720957226.X [P].2018-0130.

[8] Editorial Department of the Journal (Summary), Zhang Chenglong (Planner/Executer). Cognitive Artificial Intelligence [J]. Entrepreneur Information, 2018, 000(007):P.15-28. 